\title{
High-temperature antiferromagnetism in Yb based heavy fermion systems proximate to a Kondo insulator
}

\author{
Shintaro Suzuki $\odot,{ }^{1, *}$ Kou Takubo $\odot,{ }^{1,}{ }^{*}$ Kentaro Kuga, ${ }^{2}$ Wataru Higemoto, ${ }^{3,4}$ Takashi U. Ito, ${ }^{3}$ Takahiro Tomita $\odot,{ }^{1}$ \\ Yasuyuki Shimura, ${ }^{5}$ Yosuke Matsumoto, ${ }^{6}$ Cédric Bareille, ${ }^{1}$ Hiroki Wadati, ${ }^{1}$ Shik Shin, ${ }^{1}$ and Satoru Nakatsuji $1,7,8,9,10, \dagger$ \\ ${ }^{1}$ Institute for Solid State Physics (ISSP), University of Tokyo, Kashiwa, Chiba 277-8581, Japan \\ ${ }^{2}$ RIKEN SPring-8 Center, Sayo, Hyogo 679-5148, Japan \\ ${ }^{3}$ Advanced Science Research Center, Japan Atomic Energy Agency, Tokai, Ibaraki 319-1195, Japan \\ ${ }^{4}$ Department of Physics, Tokyo Institute of Technology, Meguro, Tokyo 152-8550, Japan \\ ${ }^{5}$ Graduate School of Advanced Science and Engineering, Hiroshima University, Higashi Hiroshima 739-8530, Japan \\ ${ }^{6}$ Max Planck Institute for Solid State Research, Heisenbergstrasse 1, Stuttgart 70569, Germany \\ ${ }^{7}$ Department of Physics, Graduate School of Science, University of Tokyo, Tokyo 113-0033, Japan \\ ${ }^{8}$ CREST, Japan Science and Technology Agency (JST), 4-1-8 Honcho Kawaguchi, Saitama 332-0012, Japan \\ ${ }^{9}$ Institute for Quantum Matter and Department of Physics and Astronomy, Johns Hopkins University, Baltimore, Maryland 21218, USA \\ ${ }^{10}$ Trans-scale Quantum Science Institute, University of Tokyo, Tokyo 113-0033, Japan
}

(Received 25 March 2018; accepted 25 March 2021; published 24 May 2021)

\begin{abstract}
Given the parallelism between the physical properties of $\mathrm{Ce}$ - and Yb-based magnets and heavy fermions due to the electron-hole symmetry, it has been rather odd that the transition temperature of the Yb-based compounds is normally very small, as low as $\sim 1 \mathrm{~K}$ or even lower, whereas Ce counterparts may often have the transition temperature well exceeding $10 \mathrm{~K}$. Here, we report our experimental discovery of the transition temperature reaching $20 \mathrm{~K}$ in a $\mathrm{Yb}$-based compound at ambient pressure. The $\mathrm{Mn}$ substitution at the $\mathrm{Al}$ site in an intermediate valence state of $\alpha-\mathrm{YbAlB}_{4}$ not only induces antiferromagnetic transition at a record high temperature of $20 \mathrm{~K}$ but also transforms the heavy-fermion liquid state in $\alpha-\mathrm{YbAlB}_{4}$ into a highly resistive metallic state proximate to a Kondo insulator.
\end{abstract}

DOI: 10.1103/PhysRevResearch.3.023140

\section{INTRODUCTION}

Correlated electron systems have provided a number of nontrivial phenomena including quantum criticality, unconventional superconductivity, and exotic magnetic/electronic orders. Specifically, $4 f$-electron systems have provided prototypical materials to study quantum criticality that appears as a result of the competition between the RKKY interaction and the Kondo effect [1-11]. $4 f$-electron systems also provide ideal platforms to investigate the effects of correlation in the presence of the strong spin-orbit coupling. The discovery of novel topological phases in correlated electron systems such as topological insulators and Weyl semimetals progressively attracted attention to even more strongly correlated system [12-17], including $4 f$-electron systems with, for example, the so-called topological Kondo insulators [18-21].

Among all lanthanide ions, most studies have focused on $\mathrm{Ce}$ - and $\mathrm{Yb}$-based compounds as they are at the one-

\footnotetext{
*These authors contributed equally to this work.

${ }^{\dagger}$ To whom correspondence should be addressed: satoru@phys.s.utokyo.ac.jp

Published by the American Physical Society under the terms of the Creative Commons Attribution 4.0 International license. Further distribution of this work must maintain attribution to the author(s) and the published article's title, journal citation, and DOI.
}

electron and the one-hole limits of the $4 f$ shell, respectively. This electron-hole symmetry implies a parallelism between the physical properties of $\mathrm{Ce}$ - and $\mathrm{Yb}$-based magnets and heavy fermions. However, the transition temperature of the Yb-based compounds is normally very small, as low as $\sim 1 \mathrm{~K}$ or even lower [3,5,22-31], whereas Ce counterparts may have the transition temperature often well exceeding $10 \mathrm{~K}$ [32-36].

Among a number of Yb-based heavy-fermion systems, $\beta$-YbAlB 4 is particularly interesting as it is the first example of a heavy-fermion superconductor and unconventional quantum criticality without tuning, namely, at ambient pressure and at zero field [8,37-39]. In contrast, its isomorphic compound $\alpha-\mathrm{YbAlB}_{4}$ has a Fermi-liquid ground state while it exhibits almost the same Kondo-lattice-like behavior at higher temperatures [40]. Recent study revealed that a small amount of Fe doping of $1.4 \%$ induces nearly the same type of quantum criticality as found in $\beta-\mathrm{YbAlB}_{4}$ at ambient pressure [41]. The clear anomaly in the low-temperature valence indicates quantum valence transition as the most likely origin [41-43].

Another particularly striking feature found in these compounds is the unusually high transition temperature of their magnetism. For example, the transition temperature exceeding $30 \mathrm{~K}$ was observed for $\beta-\mathrm{YbAlB}_{4}$ under high pressure, which is a record high transition temperature for $\mathrm{Yb}$ based compounds to date $[44,45]$. In addition, for both $\alpha$ and $\beta$ phases of $\mathrm{YbAlB}_{4}$, a sufficient Fe doping stabilizes an antiferromagnetic ordering whose transition temperature becomes as high as 


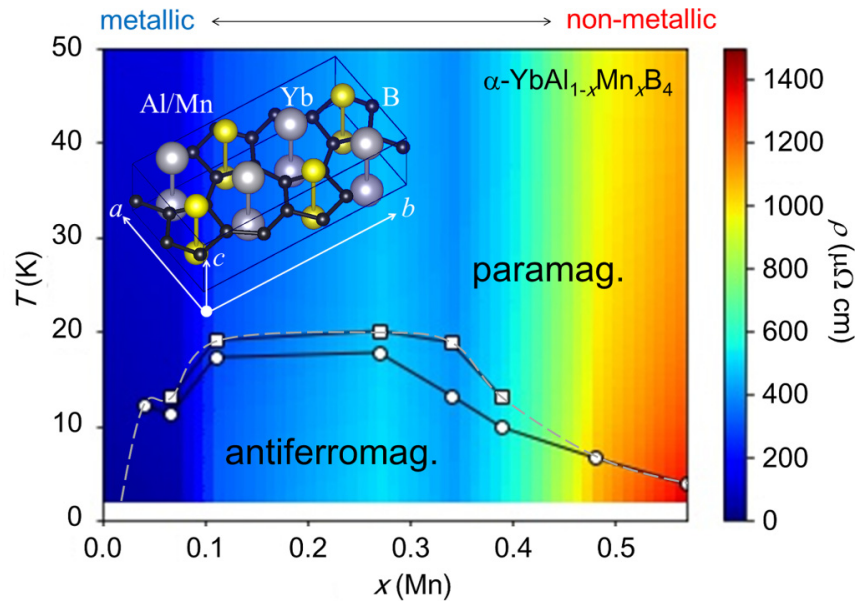

FIG. 1. Phase diagram as a function of temperature $T$ (vertical axis) and Mn concentration $x$ (horizontal axis) for $\alpha-\mathrm{YbAl}_{1-x} \mathrm{Mn}_{x} \mathrm{~B}_{4}$. The contour plot of the $a b$-plane resistivity $\rho$ is also provided in the same region of $T$ and $x$. Each point indicates the Néel temperature $T_{\mathrm{N}}$ determined by the anomaly found in the temperature dependence of the magnetization (squares) and specific heat (circles). The broken line schematically refers to a phase boundary due to the antiferromagnetic transition. Inset indicates the unit-cell crystal structure of $\alpha-\mathrm{YbAl}_{1-x} \mathrm{Mn}_{x} \mathrm{~B}_{4}$.

$10 \mathrm{~K}$ at ambient pressure $[41,46,47]$. These fascinating observations of the unusually high transition temperature as well as the unconventional quantum criticality in these systems indicate a novel mechanism behind the phenomena. A significant question would be whether the transition temperature may become even higher than $10 \mathrm{~K}$ by another type of chemical doping to these systems. If so, such a study would not only find the highest ever magnetic transition temperature in the Yb-based compounds at ambient pressure, but it will also allows further in-depth study to elucidate the electronic state change across the transition by spectroscopic method, which has been otherwise impossible for $\mathrm{Yb}$ compounds to date.
Here, we report the discovery of a high Néel temperature reaching $20 \mathrm{~K}$, the highest temperature among the Yb-based intermetallic compound at ambient pressure. As we show in Fig. 1, the Mn substitution at the Al site of $4 \%$ is enough to induce an antiferromagnetic transition in $\alpha-\mathrm{YbAlB}_{4}$. The transition temperature exceeds $20 \mathrm{~K}$ at the doping level of $\sim 30 \% \mathrm{Mn}$. The high transition temperature cannot be explained by the RKKY interaction and thus points to an itinerant type magnetic order. On the other hand, the combination of the transport and the photoemission spectroscopy (PES) measurements reveals that the Mn doping induces a hybridization gap at the Fermi energy $\left(E_{\mathrm{F}}\right)$, causing a highly resistive nonmetallic behavior near $x(\mathrm{Mn})=0.5$. Our study indicates that the extremely high transition temperature appears in a heavy-fermion (HF) state proximate to a Kondo insulator phase.

\section{RESULTS AND DISCUSSION}

Figures 2(a) and 2(b) display the temperature dependence of the $a b$-plane magnetic susceptibility and specific heat, respectively. With increasing Mn concentration $x$, the temperature dependence of the susceptibility exhibits a clear kink and a bifurcation between the zero-field-cooled (ZFC) and fieldcooled (FC) components of the magnetic susceptibility. While no magnetic anomaly is found at $x=0.01$, the bifurcation is clearly seen at the concentration $x$ higher than 0.11 , and a small anomaly appears already at $x=0.07$. Correspondingly, the specific-heat measurement finds a clear peak at almost the same temperature as the bifurcation temperature of the magnetic susceptibility curve. The anomaly observed in both the susceptibility and specific heat demonstrates that the antiferromagnetic transition is induced by the Mn substitution. Strikingly, the transition temperature $\left(T_{\mathrm{N}}\right)$ increases with increasing chemical substitution and reaches a temperature as high as $20 \mathrm{~K}$ at $x=0.27$. On the other hand, the susceptibility measured for the nonmagnetic analog $\alpha-\mathrm{LuAl}_{0.56} \mathrm{Mn}_{0.44} \mathrm{~B}_{4}$ has a much smaller value around $\chi \sim 2 \times 10^{-4} \mathrm{emu} / \mathrm{mol}$
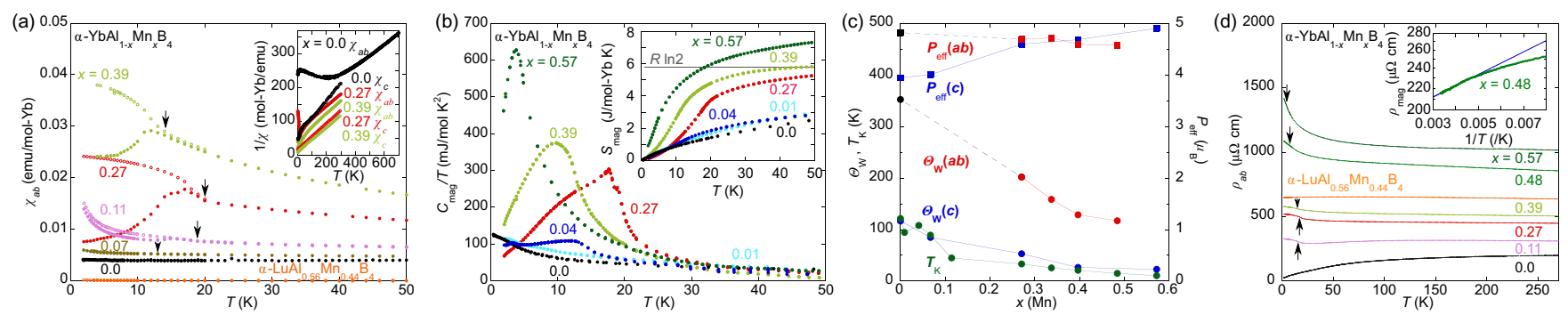

FIG. 2. (a) Temperature dependence of the magnetic susceptibility measured in the magnetic field along the $a b$ plane $(B=1000$ Oe). Open circles and filled circles refer to the field-cooled (FC) and zero-field-cooled (ZFC) components of the susceptibility, respectively. Arrows indicate the transition temperatures defined as the onset of the bifurcation between the FC and ZFC results. Inset shows temperature dependence of the inverse susceptibility. (b) Temperature dependence of the magnetic part of the specific heat divided by temperature, $C_{\text {mag }} / T . C_{\text {mag }}$ is estimated as $C_{\text {mag }}=C\left(\alpha-\mathrm{YbAl}_{1-x} \mathrm{Mn}_{x} \mathrm{~B}_{4}\right)-C\left(\alpha-\mathrm{LuAlB}{ }_{4}\right)$. Inset shows the temperature dependence of the entropy calculated using the specific heat result. (c) Substitution $x(\mathrm{Mn})$ dependence of the Weiss temperature $\left(\Theta_{\mathrm{W}}\right.$ ), the Kondo temperature $T_{\mathrm{K}}$ (left axis) and the effective moment $\left(P_{\text {eff }}\right)$ (right axis). (d) Temperature dependence of the $a b$-plane resistivity. The inset indicates the semi logarithmic plot of the $1 / T$ dependence of the magnetic part of the resistivity $\rho_{\mathrm{mag}}$ for $x=0.48$. The magnetic part $\rho_{\mathrm{mag}}$ is defined as $\rho_{\text {mag }}=\rho\left(\alpha-\mathrm{YbAl}_{0.52} \mathrm{Mn}_{0.48} \mathrm{~B}_{4}\right)-\rho\left(\alpha-\mathrm{LuAl}_{0.56} \mathrm{Mn}_{0.44} \mathrm{~B}_{4}\right)$. Black arrows indicate the transition temperature estimated by the anomaly in the temperature derivative of the resistivity and/or the peak of the specific heat. The solid line indicates the fit to the activation law with the gap $\Delta=50 \mathrm{~K}$ (see text). 
and its temperature dependence does not exhibit any anomaly. All these results including a $\mu$ SR measurement (given in the Appendix B) indicate that $\mathrm{Mn}$ in $\alpha-\mathrm{LuAl}_{0.56} \mathrm{Mn}_{0.44} \mathrm{~B}_{4}$ should be nonmagnetic.

To our knowledge, the antiferromagnetic transition temperature reaching $20 \mathrm{~K}$ is the highest among the Yb-based magnets and $\mathrm{HF}$ compounds at ambient pressure. Interestingly, $T_{\mathrm{N}}$ peaks at $x=0.27$ and starts decreasing with further substitution as found in both magnetic susceptibility and specific heat results [Figs. 2(a) and 2(b)]. It should be also noted that the Mn substitution changes the magnetic anisotropy. Whereas the pure $\alpha-\mathrm{YbAlB}_{4}$ has the Ising-type moment along the $c$ axis and the $a b$-plane susceptibility is almost temperature independent below $300 \mathrm{~K}$, the Mn substitution induces a more isotropic behavior with a strong temperature dependence in the $a b$ plane, as visible in the inverse susceptibility in the inset of Fig. 2(a).

To further understand the Mn substitution effects, we fitted both the $a b$-plane and the $c$-axis components of the susceptibility $\chi(T)$ using the Curie-Weiss (CW) law, $\frac{1}{\chi}=\frac{3 k_{\mathrm{B}}\left(T-\Theta_{\mathrm{W}}\right)}{N_{\mathrm{A}} P_{\mathrm{eff}}^{2} \mu_{\mathrm{B}}^{2}}$ where $k_{\mathrm{B}}, \Theta_{\mathrm{W}}, N_{\mathrm{A}}, P_{\text {eff }}$, and $\mu_{\mathrm{B}}$ refer the Boltzmann constant, the Weiss temperature, the Avogadro's number, the effective moment, and the Bohr magneton, respectively. The fitting was performed in the temperature range from $150 \mathrm{~K}$ to $250 \mathrm{~K}$ except for the $a b$-plane component of the pure system [Fig. 2(a) inset]. On the other hand, the magnetic part of the entropy $S_{\text {mag }}$ was estimated by $S_{\text {mag }}=\int \frac{C_{\text {mag }}}{T} d T$, assuming a linear increase of $C_{\text {mag }}$ between 0 and $2 \mathrm{~K}$. Here, $C_{\mathrm{mag}}$ indicates the magnetic part of the specific heat estimated as noted in the caption of Fig. 2(b). Interestingly, the entropy in the paramagnetic state, e.g., at $40 \mathrm{~K}$, increases with increasing Mn substitution and reaches a value of about $R \ln 2$ for $x \geqslant 0.27$ [Fig. 2(b) inset]. This indicates that the ground state remains a doublet, but the associated Kondo temperature $T_{\mathrm{K}}$ decreases significantly with increasing Mn substitution as we discuss in the following.

Figure 2(c) shows the $x(\mathrm{Mn})$ dependence of the Kondo temperature $T_{\mathrm{K}}$, of the Weiss temperature $\Theta_{\mathrm{W}}$, and of the effective moment $P_{\text {eff }} . T_{\mathrm{K}}$ was estimated from the temperature dependence of the entropy [Fig. 2(b) inset] using the relation $S_{\mathrm{mag}}\left(T=T_{\mathrm{K}} / 2\right)=(R / 2) \ln 2$. Both the Kondo temperature $T_{\mathrm{K}}$ and the Weiss temperature $\Theta_{\mathrm{W}}(c)$, extracted from the $c$-axis component of the susceptibility, nearly collapse with increasing $x(\mathrm{Mn})$. In its $a b$-plane component, the susceptibility of the pure system $(x=0)$ exhibits a peak at about $200 \sim 250 \mathrm{~K}$ due to the significant valence fluctuations in this system $[40,43,48]$. Therefore, we extended our susceptibility measurements up to $700 \mathrm{~K}$ to find a $\mathrm{CW}$ behavior above $300 \mathrm{~K}$ [Fig. 2(a) inset]. While both $T_{\mathrm{K}}$ and $\Theta_{\mathrm{W}}(c)$ are found to be of about $\sim 130 \mathrm{~K}, \Theta_{\mathrm{W}}(a b)$ reaches about $350 \mathrm{~K}$ in the pure system from the fitting of the $a b$-plane component of the susceptibility in between $600 \mathrm{~K}$ and $700 \mathrm{~K}$. With increasing $x(\mathrm{Mn})$, we find a systematic decrease in all $\Theta_{\mathrm{W}}(a b)$, $\Theta_{\mathrm{W}}(c)$, and $T_{\mathrm{K}}$. This indicates the valence fluctuation regime in $\alpha-\mathrm{YbAlB}_{4}$ is suppressed by the Mn substitution. In fact, the local moment $P_{\text {eff }}$ gradually increases to reach $4.5 \sim 4.7 \mu_{B}$ at $x>0.27$, a value equivalent to the one of the isotropic $\mathrm{Yb}^{3+}$ ion: $4.54 \mu_{B}$. Thus the Mn substitution leads the $\mathrm{Yb}$ ions to a trivalent configuration.
Generally, in the Kondo regime, the magnetic couplings are dominated by the RKKY interaction. Theoretically, the magnetic transition temperature due to the RKKY interaction is known to be proportional to the de Gennes factor [49] (see the Appendix C). However, $T_{\mathrm{N}}$ of $\alpha-\mathrm{YbAl}_{0.73} \mathrm{Mn}_{0.27} \mathrm{~B}_{4}$ is strikingly one order of magnitude higher than those with other $R \mathrm{AlB}_{4}$ systems having the $\mathrm{YCrB}_{4}$ structure [50,51]. Namely, the magnetism in $\alpha-\mathrm{YbAl}_{1-x} \mathrm{Mn}_{x} \mathrm{~B}_{4}$ is not a localized RKKY magnetism, but most likely an itinerant type.

An important effect to understand the origin of the itinerant magnetism is the increase in the hybridization strength with the Mn substitution. The substitution decreases the volume of the unit cell (given in the Appendix D), and thus applies chemical pressure to the system. The valence change discussed above from the larger $\mathrm{Yb}^{2+}$ to smaller $\mathrm{Yb}^{3+}$ ions also causes a similar trend. Therefore, the bond lengths between $\mathrm{Yb}$ and $\mathrm{B}$ become shorter, and thus the hybridization strength should increase with the Mn substitution [52,53]. Further evidence for the itinerant magnetism will be discussed below using the results obtained in the resistivity and PES measurements.

Recent structure analysis using powder $\mathrm{x}$-ray diffraction under high pressure on $\beta$-YbAlB 4 indicates that the $\mathrm{B}$ rings sandwiching $\mathrm{Yb}$ ion (Fig. 1 inset) lowers its symmetry from nearly 7 fold by application of pressure [54]. One theoretical approach pointed out that this 7-fold symmetry stabilizes the $\mathrm{Yb}$ ground state to $\mid J_{z}= \pm 5 / 2>$. This orbital character is shown to imply an anisotropic hybridization as well as the Ising behavior of the magnetic moment along the $c$ axis at ambient pressure [55,56]. These results suggest that the deformation of the local symmetry under pressure suppresses the Ising-like behavior and induces the $30-\mathrm{K}$ transition under $8 \mathrm{GPa}$ in $\beta$ - $\mathrm{YbAlB}_{4}$.

This may be also the case in $\alpha-\mathrm{YbAlB}_{4}$ and the magnetism induced by the Mn substitution. Nearly the same temperature dependence of the susceptibility has been seen for both phases of $\mathrm{YbAlB}_{4}$ at ambient pressure. Thus, the crystal electric field (CEF) scheme must be nearly the same for both phases [40]. Indeed, a recent measurement of X-ray magnetic circular dichroism indicates the ground state of $\alpha-\mathrm{YbAlB}_{4}$ to be the $\mid J_{z}= \pm 5 / 2>$ state [48]. On the other hand, the systematic change in anisotropy of the susceptibility as visible in the inset of Fig. 2(a) and in Fig. 2(c) demonstrates that the CEF ground state changes with the Mn substitution, while keeping a doublet configuration as the magnetic entropy at $40 \mathrm{~K}$ remains around $R \ln 2$. Thus, most likely the ground doublet departs from the $\left|J_{z}= \pm 5 / 2\right\rangle$ state due to the local symmetry deformation around the $\mathrm{Yb}$-site, itself induced by the $\mathrm{Mn}$ substitution. This change of ground state plays an important role in the high-temperature magnetism, as discussed below.

Another effect of the chemical substitution is the carrier doping. Such carrier doping may well change the transport properties, and therefore, we performed the longitudinal resistivity measurements [Fig. 2(d)]. The magnitude as well as the temperature dependence of the resistivity dramatically change with the Mn substitution. The good metallic behavior observed in the pure system is already lost at $x=0.11$ and the resistivity at both room temperature and $2 \mathrm{~K}$ increases nearly linearly with $x$. The resistivity at $2 \mathrm{~K}$ for $x=0.57$ is $\sim 60$ times larger than the one for $x=0$. Moreover, the temperature dependence systematically changes into a more incoherent 

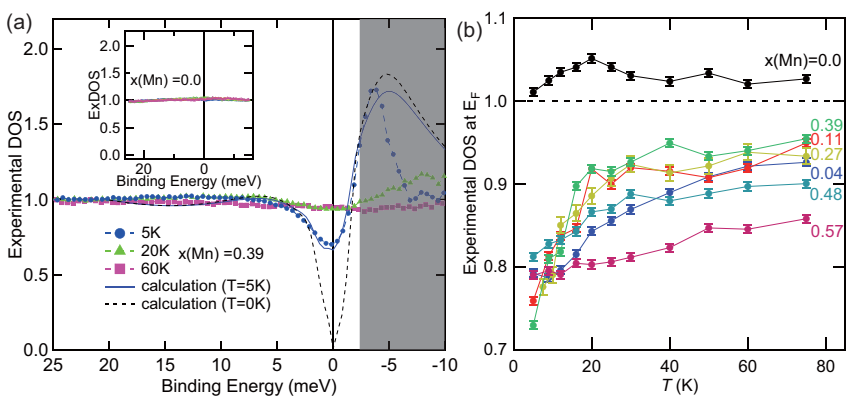

FIG. 3. (a) Experimental density of state (ExDOS) near $E_{\mathrm{F}}$ for $\alpha-\mathrm{YbAl}_{1-x} \mathrm{Mn}_{x} \mathrm{~B}_{4}(x=0.39)$ derived from the PES measurements. The dotted lines indicate the calculation based on the periodic Anderson model [61]. The uncertain energy region on ExDOS over $\sim 5 k_{\mathrm{B}} T$ above $E_{\mathrm{F}}[60,61]$ is shaded (over $\sim 2.2 \mathrm{meV}$ at $5 \mathrm{~K}$ ). Inset shows ExDOS for $x=0.0$. ExDOS for other $x$ is displayed in the Fig. 5 of Appendix. E. (b) Temperature dependence of ExDOS at $E_{\mathrm{F}}$ for various compositions.

semimetallic or nonmetallic behavior with Mn doping. These indicate that the carrier concentration must decrease with increasing Mn concentration. We also point out that the hightemperature resistivity for $x \sim 0.5$ can be roughly fitted with the activation law, namely, $\rho=\rho_{0} \exp (\Delta / T)$ with an activation energy of about $\Delta \sim 50 \mathrm{~K}$. The inset of Fig. 2(d) exemplifies such a fit with the magnetic part of the resistivity for $x=0.48$ in which the activation law is observed at high temperature from $200 \mathrm{~K}$ to above $300 \mathrm{~K}$. This suggests that the highly resistive transport induced by Mn substitution results from the system approaching an insulating state. The deviation from the activation law at low temperature indicates the presence of impurity bands which cause the saturation of the resistivity on cooling. In addition, a clear anomaly at $T_{\mathrm{N}}$ can be found for the high $T_{\mathrm{N}}$ samples with $x=0.11$ and 0.27. $\mathrm{Ce}\left(\mathrm{Ru}_{0.85} \mathrm{Rh}_{0.15}\right)_{2} \mathrm{Si}_{2}$ exhibits a quite similar behavior at a spin-density-wave (SDW) transition [57]. This suggests the SDW transition as the origin of the anomaly seen between $0.04 \leqq x \leqq 0.27$.

To investigate the origin of the insulating behavior, the density of states (DOS) near $E_{\mathrm{F}}$ was examined using the high-resolution and bulk-sensitive laser PES [58,59]. In order to evaluate the DOS below and slightly above $E_{\mathrm{F}}$, the PES spectra were divided by spectra measured on a gold reference at each measured temperature. We should stress that this method is accurate only up to $5 k_{\mathrm{B}} \mathrm{T}$ above $E_{\mathrm{F}}$ [60], as indicated by the shaded energy range of Fig. 3(a). The derived results, referred as experimental DOS (ExDOS), scarcely depends on the temperature for $x=0$ as shown by the inset of Fig. 3(a). On the other hand, for $x=0.39$, ExDOS near $E_{\mathrm{F}}$ is mostly flat at $T=75 \mathrm{~K}$ and gets suppressed as the temperature decreases. For quantitative discussion, ExDOS at $E_{\mathrm{F}}$ is plotted for each measured doping in Fig. 3(b) as a function of temperature. For $x=0$, ExDOS at $E_{\mathrm{F}}$ exceeds 1, indicating a metallic state, in agreement with previous angle resolved PES measurements [62]. In contrast, ExDOS at $E_{\mathrm{F}}$ for the doped samples $(x>0)$ falls below 1 and decreases when the temperature is lowered. The temperature-dependent depression of ExDOS is observed for all of the doped samples and starts far above the magnetic transition temperature $T_{\mathrm{N}}$, although the spectral change appears larger around $T \sim 15 \mathrm{~K}$. These results are consistent with the resistivity measurements and indicate the formation of a pseudo-gap, or a $c$ - $f$ hybridization gap, for $x>0$. One may notice that, for $x=0.39$, ExDOS across $E_{\mathrm{F}}$ is highly asymmetric and finite $(>0)$ at $E_{F}$ even at $T=5 \mathrm{~K}$. A theoretical calculation based on the periodic Anderson model with $c-f$ hybridized bands $[63,64]$ can reproduce such DOS near $E_{\mathrm{F}}$ at $T=5 \mathrm{~K}$ as also plotted in Fig. 3(a) [61]. In addition, DOS at $E_{\mathrm{F}}$ reaches 0 in the calculation for $T=0 \mathrm{~K}$ using the same parameters set for $T=5 \mathrm{~K}$, without any experimental broadening factor. This theoretical modeling suggests the gap formation observed with Mn doping to emerge from a Kondolike hybridization. Since the gap opens over $E_{\mathrm{F}}$, it naturally relates to the systematic increase of resistivity and pictures a crossover from an intermediate valence metal to a Kondo insulator as $\mathrm{Al}$ is substituted with $\mathrm{Mn}$. Such Kondo-like hybridization further implies that the $\mathrm{Yb} 4 f$-electron bands around $E_{\mathrm{F}}$ directly contribute to the transport and thus to the itinerant magnetism of $\alpha-\mathrm{YbAl}_{1-x} \mathrm{Mn}_{x} \mathrm{~B}_{4}$. The spectral shape and gap formation observed in ExDOS are further discussed in the Appendix E [61].

While Kondo insulators are commonly found in cubic systems, some orthorhombic systems, of symmetry similar to the present one, are known to be Kondo insulators [35,65-69]. In particular, $\mathrm{Ce} r_{2} \mathrm{Al}_{10}$ ( $\left.T r=\mathrm{Os}, \mathrm{Ru}\right)$ also shows a high magnetic transition temperature which motivated intensive studies to reveal its origin [36,70]. The similar highly resistive state, proximate to a Kondo insulator, found in $\alpha-\mathrm{YbAl}_{1-x} \mathrm{Mn}_{x} \mathrm{~B}_{4}$ suggests that the same type of mechanism is at the origin of the high-temperature magnetism.

Overall results indicate that the high-temperature magnetism in this system is the itinerant SDW-type induced by chemical substitution, similar to the Rh substitution case in $\mathrm{CeRu}_{2} \mathrm{Si}_{2}$ [57]. The hybridization strength increases with the Mn substitution, while the suppression of the valence fluctuations in the Kondo regime-with an almost trivalent $\mathrm{Yb}$ configuration-leads to the decreases of the Kondo and Weiss temperatures. Theoretically, the strong hybridization may lead to a SDW-type order near the onset of a Kondo insulating phase [71]. The sharp coherent feature in PES seen only for the high $T_{\mathrm{N}}$ samples between $x=0.11$ and 0.39 [61] may result from the nesting across $E_{\mathrm{F}}$ leading to a SDW-order.

The itinerant $4 f$ electrons in pure $\alpha-\mathrm{YbAlB}_{4}$ form quasitwo-dimensional bands. They do not contribute much to transport along the $c$ axis but do mainly in the $a b$ plane [40]. This suggests that the SDW-type antiferromagnetism is formed with the nesting vector in the $a b$ plane. The suppression of the magnetic anisotropy found in the susceptibility measurements by Mn doping indicates that the CEF, which initially implies the Ising-like ground state along the $c$ axis of the pure system, is modified by the distortion of the 7-fold local symmetry at the $\mathrm{Yb}$ site. As a result, the $4 f$ magnetic moment may develop a component in the $a b$ plane. As well known for the spin-flop transition in Cr [72], the SDW energy scale can be affected by the spin configuration and by its nesting vector. Therefore, the high $T_{\mathrm{N}}$ induced by $\mathrm{Mn}$ substitution might also arise from the change in the CEF scheme. Future studies of the CEF scheme, both experimental (e.g., neutron scattering, NMR measurement) and theoretical, are necessary to clarify such possible mechanism. 


\section{CONCLUSIONS}

To conclude, through the comprehensive measurements of $\alpha-\mathrm{YbAl}_{1-x} \mathrm{Mn}_{x} \mathrm{~B}_{4}$, we found that the $\mathrm{Mn}$ substitution induces a high-temperature antiferromagnetism, whose transition temperature reaches $20 \mathrm{~K}$ at $x=0.27$. This is the highest magnetic transition temperature among the Yb based HF systems at ambient pressure. Our transport measurements show that the system exhibits a high resistivity with the Mn substitution. Thanks to PES, we further highlight the formation of a gap at the Fermi level, together with a coherent feature, consistent with the resistivity measurements. These observations evidence that the Mn substitution results in an increase of the Kondo-like hybridization, driving the system out of the mixed valence state of the pure $\alpha-\mathrm{YbAlB}_{4}$ to a more localized Kondo HF state. Additionally, the onset of the gap formation in the PES measurements appears at temperature well above the magnetic transition temperature; it lifts any ambiguity about the origin of the gap and underlines the crossover from a heavy Fermi liquid to a Kondo insulator with increasing Mn doping.

\section{ACKNOWLEDGMENTS}

We thank K. Sone, C. Broholm, S. Wu, M. Suzuki, and R. Arita for useful discussions. This work is partially supported by CREST (JPMJCR18T3), PRESTO (JPMJPR15N5), Japan Science and Technology Agency, by Grants-in-Aids for Scientific Research on Innovative Areas (No. JP15H05882 and No. JP15H05883) from the Ministry of Education, Culture, Sports, Science, and Technology of Japan, and by Grants-in-Aid for Scientific Research (No. JP25707030, No. JP25887015, No. JP15J08663, No. JP16H02209, No. JP16H06345, and No. JP19H00650) from the Japanese Society for the Promotion of Science (JSPS). The use of the facilities of the Materials Design and Characterization Laboratory at the Institute for Solid State Physics, The University of Tokyo, is acknowledged. S. Suzuki was supported by Japan Society for the Promotion of Science through Program for Leading Graduate Schools (MERIT). This research is funded in part by a QuantEmX Grant from ICAM, the Gordon and Betty Moore Foundation through Grant No. GBMF5305 and by Canadian Institute for Advanced Research. S. Suzuki greatly appreciates the hospitality of Department of Physics and Astronomy of Johns Hopkins University, where a part of this work was conducted. Institute for Quantum Matter, an Energy Frontier Research Center was funded by DOE, Office of Science, Basic Energy Sciences under Award No. DE-SC0019331.

\section{APPENDIX A: MATERIALS AND METHODS}

Single crystals of $\alpha-\mathrm{YbAl}_{1-x} \mathrm{Mn}_{x} \mathrm{~B}_{4} \quad$ and $\alpha-\mathrm{LuAl}_{0.56} \mathrm{Mn}_{0.44} \mathrm{~B}_{4}$ were synthesized by an Al-flux method [73]. These were confirmed to have the same structure as $\alpha-\mathrm{YbAlB}_{4}$ by the powder X-ray diffraction measurements. The resistivity and specific-heat measurements were performed with a commercial physical property measurement system (Quantum Design, PPMS) at temperatures above $2 \mathrm{~K}$. The magnetic susceptibility measurements were made with the commercial magnetic property measurement system (Quantum Design, MPMS). All the measurements were made in the temperature range of 2 to $300 \mathrm{~K}$ (partly 2 to $700 \mathrm{~K}$ ). The Mn concentration $x$ was estimated by Inductively Coupled Plasma Atomic Emission Spectroscopy (ICP-AES) within $0.6 \%$ resolution. The $\mu \mathrm{SR}$ measurement was performed in the GPS spectrometer at the Paul Scherrer Institute, Switzerland.

The density of states near the Fermi energy $\left(E_{\mathrm{F}}\right)$ was examined using the high-resolutional and bulk-sensitive laser PES. The samples were fractured in situ perpendicular to the $c$ axis under the base pressure of $2 \times 10^{-11}$ Torr, and the spectra were recorded using a VG Scienta R4000 analyzer. The incident light was the 6th harmonic $(6.994 \mathrm{eV})$ of a $\mathrm{Nd}: \mathrm{YVO}_{4}$ quasi-cw laser (Spectra-Physics Vanguard) [58,59] and the energy resolution was set to be $1 \mathrm{meV}$. $E_{\mathrm{F}}$ was calibrated using the Fermi cutoff of an evaporated $\mathrm{Au}$. The temperature dependence of the valence band was recorded in the transmission mode at the normal incidence, while the band dispersion was barely observed in the angular-resolved mode. By employing $6.994 \mathrm{eV}$ photons, photoelectrons are detected from $E_{\mathrm{F}}$ to the binding energy of $\sim 1 \mathrm{eV}$, and from the surface to the electron escape depth of $\sim 100 \AA$ [59]. Thus, the surface contributions are expected to be very small. To evaluate the density of state (DOS), the PES spectra were divided by the spectra of gold for each temperature. This procedure is widely accepted as a method to evaluate experimental DOS (ExDOS) near $E_{\mathrm{F}}$ and it was known that this allows one to recover the thermally occupied states up to $\sim 5 k_{\mathrm{B}} T$ above $E_{\mathrm{F}}$ [60].

\section{APPENDIX B: $\mu$ SR MEASUREMENT}

To confirm the nonmagnetic state, we have carried out the muon spin-relaxation measurements under zero-magnetic field $(\mathrm{ZF}-\mu \mathrm{SR})$ in a sample of randomly oriented single crystals of $\alpha-\mathrm{LuAl}_{0.56} \mathrm{Mn}_{0.44} \mathrm{~B}_{4}$ down to $1.7 \mathrm{~K}$. Figure 4(a) shows the temperature dependence of the $\mathrm{ZF}-\mu \mathrm{SR}$ spectra in $\alpha$-LuAl ${ }_{0.56} \mathrm{Mn}_{0.44} \mathrm{~B}_{4}$ at $50 \mathrm{~K}$ and $1.7 \mathrm{~K}$. We have observed no significant change in the ZF- $\mu \mathrm{SR}$ spectra below $50 \mathrm{~K}$. Time evolution of the muon decay asymmetry $A(t)$ were analyzed by using the Kubo-Toyabe function,

$$
A(t)=A(0)\left[\frac{1}{3}+\frac{2}{3}\left(1-\Delta^{2} t^{2}\right) \exp \left(-\frac{\Delta^{2} t^{2}}{2}\right)\right] .
$$

Here, $\Delta$ is the static relaxation rate due to the distribution of internal fields with dipolar width $\Delta / \gamma \mu(\gamma \mu$ is the muon gyromagnetic ratio). The temperature dependence of $\Delta$ is also shown in the inset of Fig. 4(a). The size in $\Delta$ is so small that the origin of the internal field at the muon site should be considered due to a weak and static magnetic field from the nuclear dipolar moments of ${ }^{11} \mathrm{~B},{ }^{55} \mathrm{Mn},{ }^{175} \mathrm{Lu}$, and we have not observed any additional internal field down to $1.7 \mathrm{~K}$. Thus, it is reasonable to conclude that the magnetic state of $\alpha-\mathrm{LuAl}_{0.56} \mathrm{Mn}_{0.44} \mathrm{~B}_{4}$ is nonmagnetic and the magnetism of $\alpha-\mathrm{YbAl}_{1-x} \mathrm{Mn}_{x} \mathrm{~B}_{4}$, is not owing to $\mathrm{Mn} 3 d$, but $\mathrm{Yb} 4 f$ moments.

\section{APPENDIX C: DE GENNES FACTOR}

Theoretically, the magnetic transition temperature due to the RKKY interaction is known to be proportional to the de Gennes factor, namely, (de Gennes factor $)=$ $\left(g_{J}-1\right)^{2} J(J+1)$. In this equation, $g_{J}$ and $J$ are the Lande's 
(a)

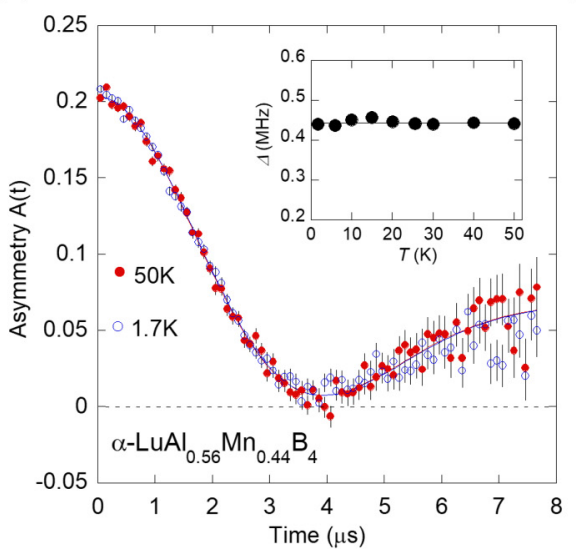

(b)

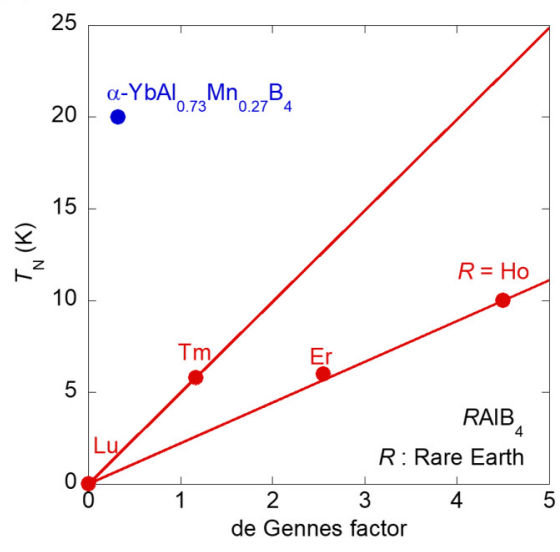

(c)

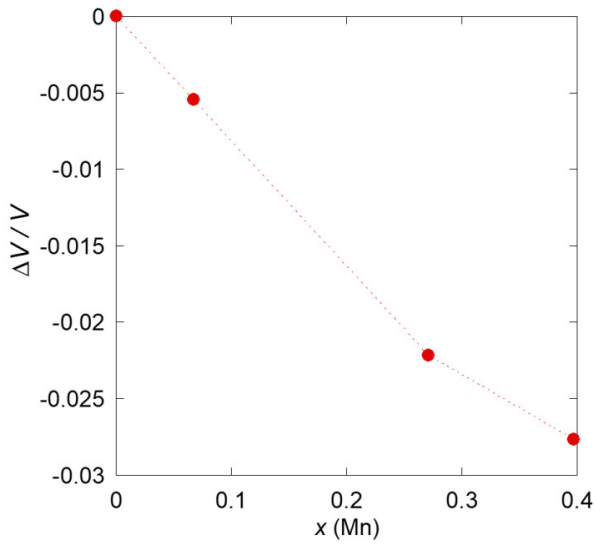

FIG. 4. (a) ZF- $\mu$ SR spectra in $\alpha-\mathrm{LuAl}_{0.56} \mathrm{Mn}_{0.44} \mathrm{~B}_{4}$ at $50 \mathrm{~K}$ and $1.7 \mathrm{~K}$. Inset shows temperature dependence of the static relaxation rate $\Delta$ in $\alpha-\mathrm{YbAl}_{1-x} \mathrm{Mn}_{x} \mathrm{~B}_{4}$. No significant change of $\Delta$ is seen as a function of temperature. (b) de Gennes factor and the transition temperature of the $R \mathrm{AlB}_{4}$ systems with the $\mathrm{YCrB}_{4}$-type structure. ( $R$ refers to rare earth elements.) $T_{\mathrm{N}}$ of $R=\mathrm{Tm}$ and $R=\mathrm{Er}$, Ho are determined by magnetic anomaly due to the hysteresis seen in the susceptibility reported in the literature [50] and [51], respectively. Solid lines indicate the naive expectation of the transition temperatures for $R \mathrm{AlB}_{4}$ based on these transition temperatures reported for $R=\mathrm{Tm}$, Er, and Ho [50,51], assuming that the transition temperature is proportional to the de Gennes factor. (c) The Mn substitution dependence of the $\Delta V / V$, which refers to the ratio of the difference $\Delta V$ of the unit cell volume between $\alpha-\mathrm{YbAl}_{1-x} \mathrm{Mn}_{x} \mathrm{~B}_{4}$ and $\alpha-\mathrm{YbAlB}_{4}$ over the unit-cell volume $V$ of $\alpha$-YbAlB .

$g$ factor and total angular momentum of a rare earth ion respectively. In fact, this relation has been confirmed so far in various systems including $R \mathrm{Ni}_{2} \mathrm{~B}_{2} \mathrm{C}$ [74], $R \mathrm{Pd}_{2} \mathrm{~Pb}$ [75], $R A \mathrm{In}_{5}$ [76], and $R_{2} A \mathrm{In}_{8}$ [76] ( $A=\mathrm{Rh}$ or Ir).

$R \mathrm{AlB}_{4}$ with the $\mathrm{YCrB}_{4}$ structure type is known to have a magnetic ordering for $R=\mathrm{Tm}$, Er, and Ho, as summarized in Fig. 4(b). Using the de Gennes factor, one may estimate the transition temperature as shown by the solid lines in Fig. 4(b), which indicates $T_{\mathrm{N}}$ expected for $R=\mathrm{Yb}$ should be less than $2 \mathrm{~K}$. This is actually the same scale as the magnetic ordering temperatures found in other $\mathrm{Yb}$ based heavy-fermion systems [23,24]. In sharp contrast, $T_{\mathrm{N}}$ of $\alpha-\mathrm{YbAl}_{0.73} \mathrm{Mn}_{0.27} \mathrm{~B}_{4}$ is strikingly one order of magnitude higher than those with other $\mathrm{Yb}$-based HF systems and even with other $R \mathrm{AlB}_{4}$ systems, thus indicating that the magnetism in $\alpha-\mathrm{YbAl}_{1-x} \mathrm{Mn}_{x} \mathrm{~B}_{4}$ is not a localized RKKY magnetism, but most likely an itinerant type.

\section{APPENDIX D: CHEMICAL PRESSURE AND ROLES OF THE Mn SUBSTITUTION}

The Mn substitution induces two effects in $\alpha-\mathrm{YbAl}_{1-x} \mathrm{Mn}_{x} \mathrm{~B}_{4}$. One is the chemical pressure. The other is the hole doping leading to the valence change from $\mathrm{Yb}^{2+}$ to $\mathrm{Yb}^{3+}$ as discussed in the main text. First, the Mn substitution decreases the volume of the unit cell as shown in Fig. 4(c), and thus applies the chemical pressure to the system. The ionic-size change from the larger $\mathrm{Yb}^{2+}$ to smaller $\mathrm{Yb}^{3+}$ ions also causes a similar trend. Therefore, the bond lengths between $\mathrm{Yb}$ and $\mathrm{B}$ shorten with the $\mathrm{Mn}$ substitution, and thus the hybridization becomes stronger $[52,53]$. On the other hand, the $\mathrm{Yb}$ valence for $x>0.27$ reaches $3+$ and thus the valence fluctuations are mostly suppressed with the Mn substitution. In the Yb-based heavy fermions, the suppression of the valence fluctuation tends to decrease the Kondo temperature [52].

\section{APPENDIX E: PHOTOEMISSION SPECTROSCOPY}

To investigate the origin of the insulating behavior, we discuss the results of the photoemission spectroscopy (PES). The insets of Figs. 5(a)- 5(f) show the high-resolution PES spectra near $E_{\mathrm{F}}$ for $\alpha-\mathrm{YbAl}_{1-x} \mathrm{Mn}_{x} \mathrm{~B}_{4}$ at various temperatures. To evaluate DOS, the PES spectra were divided by the spectra of gold for each temperature. The derived results, namely ExDOS, are given in the main panels of Figs. 5 for $x(\mathrm{Mn})=$ (a) 0.0 , (b) 0.04 , (c) 0.11 , (d) 0.39 , (e) 0.48 , and (f) 0.57 , respectively [and in Fig. 3(a) for $x(\mathrm{Mn})=0.0$ and 0.39 of the main text]. Since PES measures the electrons in the occupied state including thermally distributed ones above $E_{\mathrm{F}}$, this procedure has been established to recover DOS above $E_{\mathrm{F}}$ up to $\sim 5 k_{\mathrm{B}} T$ [60]. The spectral features above $5 k_{\mathrm{B}} T$ may be unreliable, and then are indicated by the dashed lines in Fig. 5(a)-5(f). The derived ExDOS for $x=0.0$ scarcely depends on temperature. On the other hand, ExDOSs near $E_{\mathrm{F}}$ for all of the doped samples $(x>0.0)$ are mostly flat at $T=75 \mathrm{~K}$ and suppressed on cooling. Namely, the formation of the pseudo-gap is found for the doped samples. For the $0.11 \leqq x \leqq 0.48$ samples, the gap is highly asymmetric and the rapid growth of ExDOS is observed especially below $T \sim 15 \mathrm{~K}$, while its peak top is in the uncertain energy region $\left(5 k_{\mathrm{B}} T \sim 2.2 \mathrm{meV}\right.$ at $\left.5 \mathrm{~K}\right)$. These results indicate the appearance of coherent state of $4 f$ electrons above $E_{\mathrm{F}}$ at low temperature. For the low Mn concentration region with $x \geqq$ 0.04 , the pseudo-gap near $E_{\mathrm{F}}$ is also observed at low temperatures as shown in Fig. 5(b), corresponding to the anomalies of the specific heat at $T_{\mathrm{N}}$ [see also Figs. 1 and 2(b) of the main text]. However, the rapid increase of DOS above $E_{\mathrm{F}}$ is not observed at low temperature and the gap above $40 \mathrm{~K}$ are indistinct for $x=0.04$ compared to those found for $x \geqq 0.11$, possibly because of the low $T_{\mathrm{N}}$ and low resistivity. Furthermore, almost no shift of the chemical potential is observed in the entire doping range as shown in Figs. 5(b)- 5(f). The energy of ExDOS minimum (and the coherent state above $E_{\mathrm{F}}$ ) 
(a)

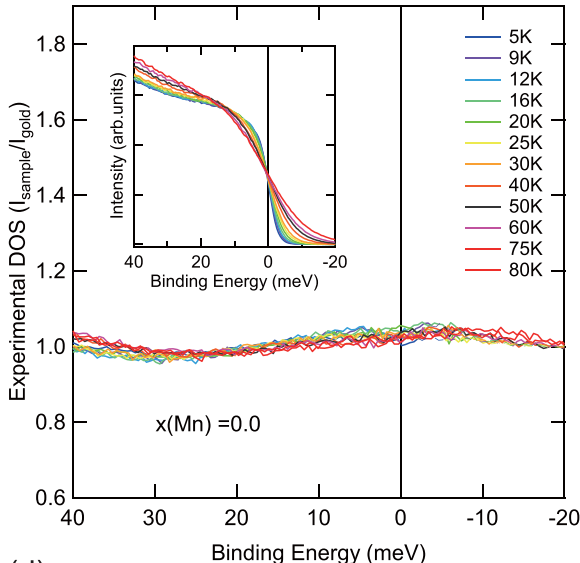

(d)

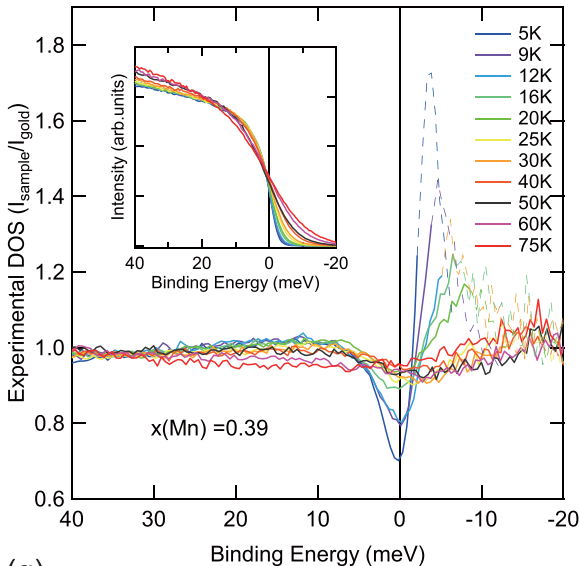

(g)

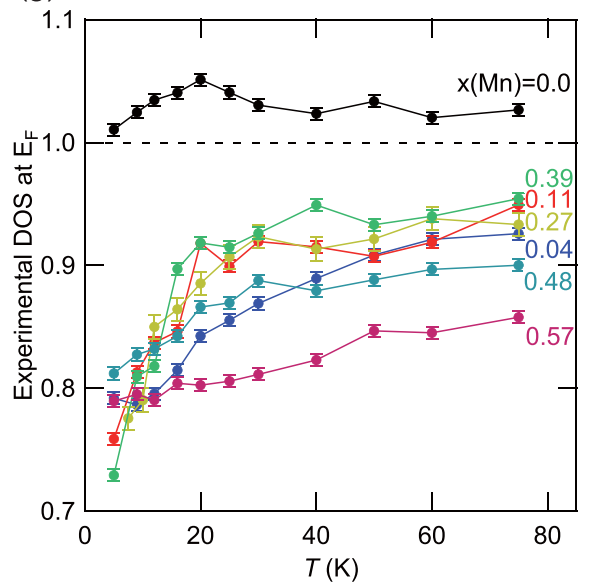

(b)

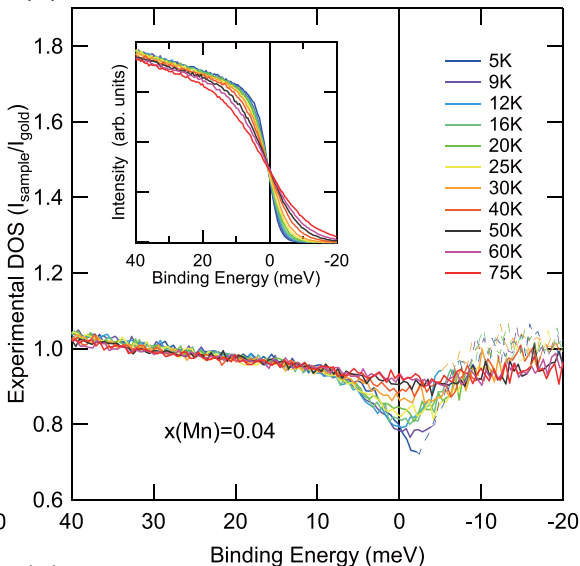

(e)

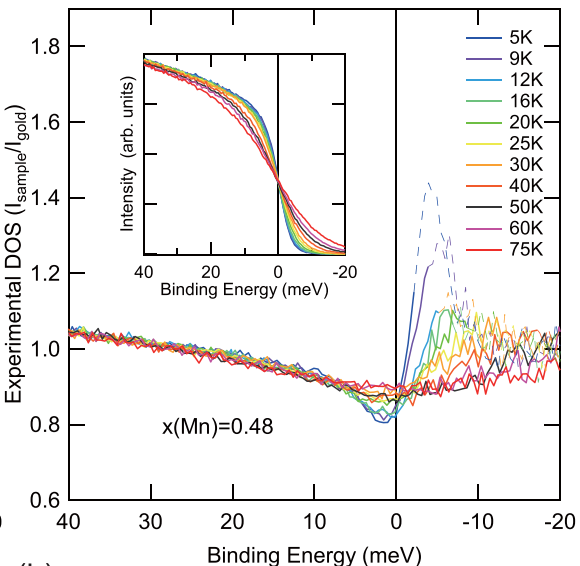

(h)

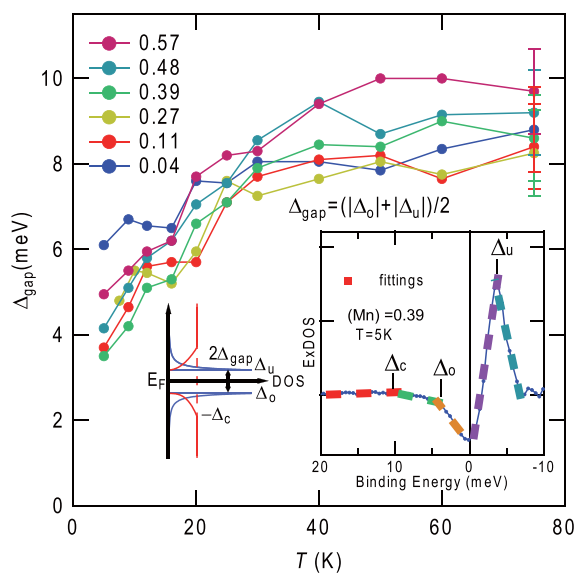

(c)

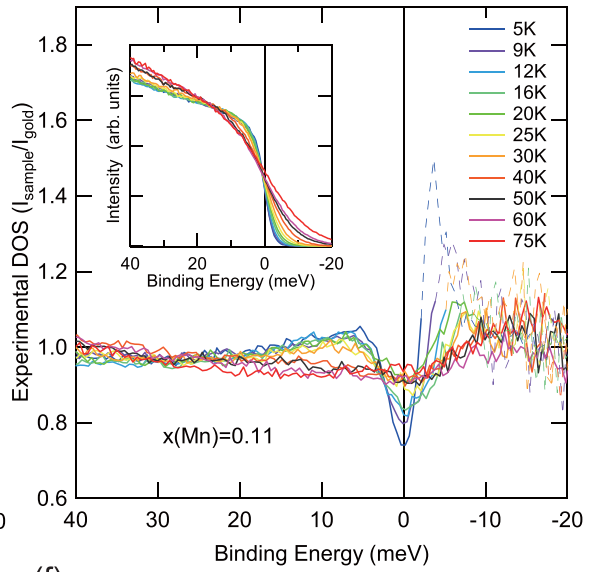

(f)

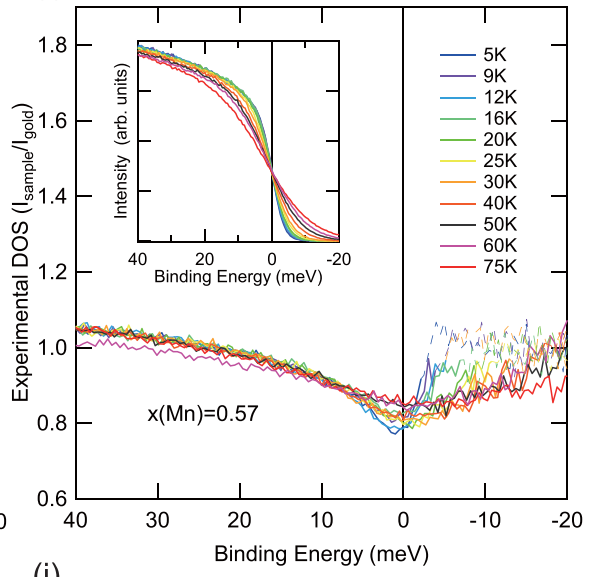

(i)

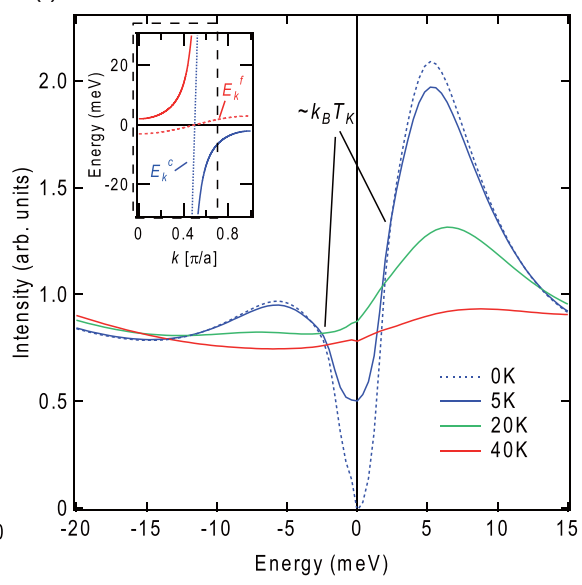

FIG. 5. [(a)-(f)] Experimental density of state (ExDOS) near $E_{\mathrm{F}}$ derived from the PES measurements for $\alpha-\mathrm{YbAl}_{1-x} \mathrm{Mn}_{x} \mathrm{~B}_{4}$ of $x(\mathrm{Mn})=$ (a) 0.0 , (b) 0.04 , (c) 0.11 , (d) 0.39 , (e) 0.48 , and (f) 0.57, respectively. ExDOSs in unreliable energy region above 5k $T$ [60] are indicated by the dashed lines. Insets show the raw PES spectra for each composition. The PES spectra are normalized by the area between -20 and $60 \mathrm{meV}$. [(g),(h)] Temperature dependence of (g) ExDOS at $E_{\mathrm{F}}$ and (h) gap size $\Delta_{\text {gap }}=\left(\left|\Delta_{\mathrm{o}}\right|+\left|\Delta_{\mathrm{u}}\right|\right) / 2$ for $\alpha$-YbAl $\mathrm{Yb}_{1-x} \mathrm{Mn}_{x} \mathrm{~B}_{4}$ estimated from the ExDOS spectra. Inset shows a schematic diagram of partial DOS in the $c$ - $f$ hybridization model and an example of the line fit for $x=0.39$ at $T=5 \mathrm{~K}$. (i) Calculated DOS based on the $c$ - $f$ hybridization model for $\alpha-\mathrm{YbAl}_{1-x} \mathrm{Mn}_{x} \mathrm{~B}_{4}[x(\mathrm{Mn})=0.39]$. The parameters are $v=100$ meV for $T=5 \mathrm{~K}$ and $v=105 \mathrm{meV}$ for $T=20 \mathrm{~K}$ and $40 \mathrm{~K}$ with $T_{\mathrm{K}}=25 \mathrm{~K}$ for $x(\mathrm{Mn})=0.39$, which is taken from the results shown in Fig. 2(c) of the main text. The dotted line denotes the calculation at $T=0 \mathrm{~K}$ using same parameters for $T=5 \mathrm{~K}$ without the experimental broadening factor. Inset shows the dispersions of the $c$ - $f$ hybridized bands assumed in the calculation. The dashed box denotes the Brillouin zone accepted in the PES measurement with $h v=7 \mathrm{eV}$. 
at the lowest temperature shifts to higher (and lower) energy, as the $x(\mathrm{Mn})$ increases. Interestingly, the ExDOS minimum is located just at $E_{\mathrm{F}}$ for high $T_{\mathrm{N}}$ compounds such as $x=0.11$ and 0.39 .

For quantitative discussion, ExDOS at $E_{\mathrm{F}}$ are plotted in Fig. 5(g) [and Fig. 3(b) of the main text] as a function of temperature. ExDOS at $E_{\mathrm{F}}$ for $x=0$ exceeds 1 , indicating the Fermi-liquid metal. On the other hand, ExDOS at $E_{\mathrm{F}}$ for the doped samples $(x>0)$ falls below 1 and decreases on cooling. The ExDOS decreases with temperature for all of the doped samples. Especially for $0.11 \leqq x \leqq 0.39$, such reduction starts above the magnetic transition temperature $T_{\mathrm{N}}$, and becomes the most notable below around $T \sim 15-20 \mathrm{~K}$ close to $T_{\mathrm{N}}$. As the spectral changes or gap formations are apparently observed above $T_{\mathrm{N}}$ [see the spectra at $T>25 \mathrm{~K}$ of $x=0.48$ or 0.57 , whose $T_{\mathrm{N}}$ is below $10 \mathrm{~K}$.], the reduction of the DOS may well start at such a high temperature as the order of the (pseudo-)gap sizes, (corresponding to $2 \Delta_{\text {gap }} \sim 10-16 \mathrm{meV}$ at $120-180 \mathrm{~K}$ estimated later) and become more evident due to the formation of the antiferromagnetic ordering. Thus, the SDW or antiferromagnetic order is a secondary effect and the $c$ - $f$ hybridization is the most likely the origin for these psuedo-gap formation. These results are further consistent with the activation-type behavior seen in the resistivity measurements, which reveals the formation of the gap of $\sim 50 \mathrm{~K}$ at the temperature around $200 \mathrm{~K}$ and above [Fig. 2(d) inset of the main text].

In addition, we determined the binding energies of the three features $\Delta_{c}, \Delta_{o}$, and $\Delta_{u}$, illustrated in the inset of Fig. $5(\mathrm{~h})$. The characteristic features of the binding energy for the $c$ - $f$ hybridization gaps were examined for various heavy-fermion systems [77-79]. According to the $c-f$ hybridization model, as the temperature decreases, the $c$ - $f$ hybridization between the dispersive conduction band and the heavy flat $\mathrm{Yb} f$ level gradually reallocate, resulting in bonding and antibonding bands below and above $E_{\mathrm{F}}$, respectively. Near $E_{\mathrm{F}}$, the heavy $\mathrm{Yb} f$-derived bands are located at $\Delta_{o}$ and $\Delta_{u}$ both sides of $E_{\mathrm{F}}$ [Inset of Fig. 5(h)]. Within the energy region where the conduction band hybridizes with the $f$ level, the conduction band loses its intensity and forms a pseudo-gap within $\Delta_{c}$ across $T_{\mathrm{K}}$. To evaluate the characteristic features of the binding energies, the ExDOS spectra were fitted by connecting 5 lines in the energy range between 20 and $-15 \mathrm{meV}$. We attribute the higher-binding-energy feature to the pseudo-gap of the conduction band $\Delta_{c}$ where the depression of DOS starts. On the other hand, the shoulder structures in the occupied and unoccupied region near $E_{\mathrm{F}}$ are attributed to $\Delta_{o}$ and $\Delta_{u}$, respectively. An example of the fitting is also shown in the inset of Fig. 5(h) for $x=0.39$ at $T=5 \mathrm{~K}$. The crossing points of the line fittings were roughly corresponding to the inflection points of the spectra, namely, the $\Delta_{c}, \Delta_{o}$, and $\Delta_{u}$ were evaluated in this procedure. The gap size, which roughly corresponds to the transport gap, can be defined as the half of them, $\Delta_{\text {gap }}=\left(\left|\Delta_{o}\right|+\left|\Delta_{u}\right|\right) / 2$ and the results are summarized in Fig. 5(h). The shoulder structures are shifted to $E_{\mathrm{F}}$ and then $\Delta_{\text {gap }}$ reduces at low temperatures for all of the doped samples. The shift of the $\mathrm{Yb} 4 f$ level to the lower binding energy side at low temperatures are also observed in the Kondo metal $\mathrm{YbAl}_{3}$ [78] and insulator $\mathrm{YbB}_{12}$ [79,80]. These behaviors were reasonably explained within the scheme of the periodic
Anderson model $[64,81,82]$. The temperature-dependent shift of the $\mathrm{Yb} 4 f$ level and depression of DOS at $E_{\mathrm{F}}$ are indicated by a calculation based on this regime. Significantly, the size of the transport gap $\Delta$ seen in the inset of Fig. 2(b) of the main text has roughly the same order of magnitude as those seen in $\Delta_{\text {gap }}$ obtained in the above analyses of the PES spectra. This reveals that the origin of the gap formation is the Kondo hybridization and thus the system at $x \sim 0.5$ should be regarded as proximate to a Kondo insulating state.

One may notice that the spectral shape of ExDOS across $E_{\mathrm{F}}$ at low temperature is highly asymmetric and much alike the "Fano lineshape" observed in scanning tunneling spectroscopy (STS) experiments for several Kondo systems [83-86]. The observation of the Fano lineshape on the STS experiments has been strong evidence of the Kondo hybridization gap for these systems. The asymmetry of DOS across $E_{\mathrm{F}}$ on the STS experiments mainly arise from the interference between the tunneling amplitude $t_{f}$ and $t_{c}$ which are the hopping amplitudes from the tip to $f$ level and conduction bands, respectively. On the other hand, in the PES process, there is no matrix element corresponding to the difference of the tunneling amplitudes in STS. However, the limitation of the Brillouin zone accepting in the PES experiments may well explain the observed gap asymmetricity. The acceptance angle of the PES analyzer is $\sim \pm 15$ degree, which corresponds to $\sim \pm 0.25 \AA^{-1}$ in the momentum space for PES with $h v=7 \mathrm{eV}(\mathrm{W} \sim 4 \mathrm{eV})$. This fact limits the PES measurement performing over $\sim 35 \%$ of the Brillouin zone (BZ) in the $a b$ plane of $\alpha-\mathrm{YbAl}_{1-x} \mathrm{Mn}_{x} \mathrm{~B}_{4}$. In the $\mathrm{Yb}$ system, the unoccupied $f$-derived band is located near $\Gamma$ point as illustrated in the inset of Fig. 5(i) and therefore enhanced in the experiment. On the other hand, the occupied $f$-derived band resides near the zone boundary. Therefore, the peak in the valence band will be much suppressed in the present low-energy PES measurements.

In the literature of the PES and STS studies for several heavy-fermion system, DOS near the hybridization gap can be modeled by the Periodic Anderson model [87]. The energies of two hybridized bands are described as

$$
E_{k}^{ \pm}=\frac{E_{k}^{c}+E_{k}^{f}}{2}+\sqrt{\left(\frac{E_{k}^{c}-E_{k}^{f}}{2}\right)^{2}+v^{2}}
$$

where $E_{k}^{c}$ and $E_{k}^{f}$ are the dispersions of the electron like conduction band and heavy flat $f$ band, respectively. and $v$ is the hybridization strength. Fig. 5(i) displays an example of the calculated DOS based on the $c$ - $f$ hybridized bands above. The dispersions and Brillouin zone assumed in the calculation are given in the inset of Fig. 5(i). In this calculation, the lifetime of the $c$ and $f$ electrons are included as the self-energy of the Green function, which is governed by the Kondo temperature $T_{\mathrm{K}}[88,89]$ and consistent with the previous angle-resolved PES study of $\mathrm{YbAlB}_{4}$ [62]. Here, the energy and momentum dependencies of the self-energy were disregarded. The peak width and line shape of the calculation are in good agreement with the experiment for $x=0.39$ as can be seen in Fig. 3(a) of the main text. Especially, the shoulder structures of $\Delta_{o}$ and $\Delta_{u}$ agree with the order of the inflection point of $\sim k_{B} T_{\mathrm{K}}$ in the 
calculation. In addition, DOS at $E_{\mathrm{F}}$ reaches 0 on the calculation for $T=0 \mathrm{~K}$ using the same parameter sets for $T=$ $5 \mathrm{~K}$ without the experimental broadening factors, indicating the ground state of $\alpha-\mathrm{YbAl}_{1-x} \mathrm{Mn}_{x} \mathrm{~B}_{4}$ is proximity to a Kondo insulator.

Because the PES cross section around $7 \mathrm{eV}$ excitation energies is rather small for the $4 f$ state of the rare earth, coherent $4 f$ bands above and below $E_{\mathrm{F}}$ usually appear rather weak in such a low-energy photoemission study for other $\mathrm{Yb}$ compounds. However, the $4 f$ states in $\alpha-\mathrm{YbAl}_{1-x} \mathrm{Mn}_{x} \mathrm{~B}_{4}$ are well hybridized with the conduction band mainly composed of the surrounding B $2 p$ orbitals, which have a large cross section for $7-\mathrm{eV}$ photons. Thus, the asymmetry of ExDOS may arise from the difference in the cross sections for the $4 f$ state and for the conduction boron bands, as well as the limitation of the acceptance zone in the PES process. The occupied bands are mainly composed of the $4 f$ state whose cross section is very small. On the other hand, the unoccupied bands are mostly composed of the intense boron state, while these two states should hybridize with each other. In fact, the band structure calculations for $\mathrm{YbAlB}_{4}$ find that the bands crossing $E_{\mathrm{F}}$ mostly arise from the $\mathrm{B} 2 p$ states and the $\mathrm{Yb} 4 f$ bands below $E_{\mathrm{F}}$ [90,91]. This is fully consistent with the formation of the hybridization gap discussed above. As the spectra do not cover the entire BZ, we need to use the resistivity measurements to see if the gap closes in BZ. Our resistivity temperature dependence finds the activation-type behavior, indicating the gap is of the order of $50 \mathrm{~K}$. This is of the same order of magnitude as the gap estimated for the hybridization gap using PES measurements [Fig. 5(h)]. This resistivity behavior indicates that it is unlikely that the gap is closed in the missing part of the BZ.

The similar coherent feature was observed in the lowenergy PES experiments for the antiferromagnetic Kondosemiconductors such as $\mathrm{CeRu}_{2} \mathrm{Al}_{10}$ and $\mathrm{CeOs}_{2} \mathrm{Al}_{10}$ [77]. On the other hand, the hump above $E_{\mathrm{F}}$ was less distinctive for the paramagnetic $\mathrm{CeFe}_{2} \mathrm{Al}_{10}$. For $\alpha-\mathrm{YbAl}_{1-x} \mathrm{Mn}_{x} \mathrm{~B}_{4}$, the coherent features above $E_{\mathrm{F}}$ on ExDOS are distinguishable only in the region having high $T_{\mathrm{N}}$ i.e., $0.11 \leqq x(\mathrm{Mn}) \leqq 0.39$. Notably, the coherent feature in this region is located very close to $E_{\mathrm{F}}$ as it can be observed in PES experiments at low temperatures. On the other hand, for $x(\mathrm{Mn})=0.57\left(T_{\mathrm{N}}<5 \mathrm{~K}\right)$, the unoccupied coherent feature are likely far above $E_{\mathrm{F}}$. For $0.11 \leqq x(\mathrm{Mn}) \leqq$ 0.39 , the steep hybridization gap can be found but very narrow at low temperature. Therefore, these results indicate that the nesting between two bands across $E_{\mathrm{F}}$ can be enhanced especially in this region and indicate the SDW-type order with high $T_{\mathrm{N}}$. Further theoretical studies are necessary to clarify this point.
[1] H. v. Löhneysen, A. Rosch, M. Vojta, and P. Wölfle, Fermiliquid instabilities at magnetic quantum phase transitions, Rev. Mod. Phys. 79, 1015 (2007).

[2] P. Gegenwart, Q. Si, and F. Steglich, Quantum criticality in heavy-fermion metals, Nat. Phys. 4, 186 (2008).

[3] Z. Fisk, P. C. Canfield, W. P. Beyermann, J. D. Thompson, M. F. Hundley, H. R. Ott, E. Felder, M. B. Maple, M. A. Lopez de la Torre, P. Visani, and C. L. Seaman, Massive Electron State in YbBiPt, Phys. Rev. Lett. 67, 3310 (1991).

[4] H. Hegger, C. Petrovic, E. G. Moshopoulou, M. F. Hundley, J. L. Sarrao, Z. Fisk, and J. D. Thompson, Pressure-Induced Superconductivity in Quasi-2D CeRhIn ${ }_{5}$, Phys. Rev. Lett. 84, 4986 (2000).

[5] P. Gegenwart, J. Custers, C. Geibel, K. Neumaier, T. Tayama, K. Tenya, O. Trovarelli, and F. Steglich, Magnetic-Field Induced Quantum Critical Point in $\mathrm{YbRh}_{2} \mathrm{Si}_{2}$, Phys. Rev. Lett. 89, 056402 (2002).

[6] H. Q. Yuan, F. M. Grosche, M. Deppe, C. Geibel, G. Sparn, and F. Steglich, Observation of two distinct superconducting phases in $\mathrm{CeCu}_{2} \mathrm{Si}_{2}$, Science 302, 2104 (2003).

[7] A. Bianchi, R. Movshovich, C. Capan, P. G. Pagliuso, and J. L. Sarrao, Possible Fulde-Ferrell-Larkin-Ovchinnikov Superconducting State in $\mathrm{CeCoIn}_{5}$, Phys. Rev. Lett. 91, 187004 (2003).

[8] S. Nakatsuji, K. Kuga, Y. Machida, T. Tayama, T. Sakakibara, Y. Karaki, H. Ishimoto, S. Yonezawa, Y. Maeno, E. Pearson et al., Superconductivity and quantum criticality in the heavy-fermion system $\beta$-YbAlB 4 , Nat. Phys. 4, 603 (2008).

[9] A. Sakai and S. Nakatsuji, Kondo effects and multipolar order in the cubic $\operatorname{PrTr}_{2} \mathrm{Al}_{20}$ (Tr= Ti, V), J. Phys. Soc. Jpn. 80, 063701 (2011).
[10] K. Matsubayashi, T. Tanaka, A. Sakai, S. Nakatsuji, Y. Kubo, and Y. Uwatoko, Pressure-Induced Heavy Fermion Superconductivity in the Nonmagnetic Quadrupolar System $\operatorname{PrTi}_{2} \mathrm{Al}_{20}$, Phys. Rev. Lett. 109, 187004 (2012).

[11] M. Tsujimoto, Y. Matsumoto, T. Tomita, A. Sakai, and S. Nakatsuji, Heavy-Fermion Superconductivity in the Quadrupole Ordered State of $\operatorname{PrV}_{2} \mathrm{Al}_{20}$, Phys. Rev. Lett. 113, 267001 (2014).

[12] M. Z. Hasan and C. L. Kane, Colloquium: Topological insulators, Rev. Mod. Phys. 82, 3045 (2010).

[13] S.-M. Huang, S.-Y. Xu, I. Belopolski, C.-C. Lee, G. Chang, B. Wang, N. Alidoust, G. Bian, M. Neupane, C. Zhang et al., A Weyl Fermion semimetal with surface Fermi arcs in the transition metal monopnictide TaAs class, Nat. Commun. 6, 7373 (2015).

[14] S.-Y. Xu, I. Belopolski, N. Alidoust, M. Neupane, G. Bian, C. Zhang, R. Sankar, G. Chang, Z. Yuan, C.-C. Lee, et al., Discovery of a Weyl fermion semimetal and topological Fermi arcs, Science 349, 613 (2015).

[15] B. Q. Lv, H. M. Weng, B. B. Fu, X. P. Wang, H. Miao, J. Ma, P. Richard, X. C. Huang, L. X. Zhao, G. F. Chen et al., Experimental Discovery of Weyl Semimetal TaAs, Phys. Rev. X 5, 031013 (2015).

[16] S. Nakatsuji, N. Kiyohara, and T. Higo, Large anomalous Hall effect in a non-collinear antiferromagnet at room temperature, Nature (London) 527, 212 (2015).

[17] K. Kuroda, T. Tomita, M.-T. Suzuki, C. Bareille, A. A. Nugroho, P. Goswami, M. Ochi, M. Ikhlas, M. Nakayama, S. Akebi et al., Evidence for magnetic Weyl fermions in a correlated metal, Nat. Mater. 16, 1090 (2017). 
[18] M. Dzero, K. Sun, V. Galitski, and P. Coleman, Topological Kondo Insulators, Phys. Rev. Lett. 104, 106408 (2010).

[19] S. Wolgast, Ç. Kurdak, K. Sun, J. W. Allen, D.-J. Kim, and Z. Fisk, Low-temperature surface conduction in the Kondo insulator $\mathrm{SmB}_{6}$, Phys. Rev. B 88, 180405(R) (2013).

[20] G. Li, Z. Xiang, F. Yu, T. Asaba, B. Lawson, P. Cai, C. Tinsman, A. Berkley, S. Wolgast, Y. S. Eo et al., Two-dimensional Fermi surfaces in Kondo insulator $\mathrm{SmB}_{6}$, Science 346, 1208 (2014).

[21] M. Neupane, N. Alidoust, S.-Y. Xu, T. Kondo, Y. Ishida, D. J. Kim, C. Liu, I. Belopolski, Y. J. Jo, T.-R. Chang et al., Surface electronic structure of the topological Kondo-insulator candidate correlated electron system $\mathrm{SmB}_{6}$, Nat. Commun. 4, 2991 (2013).

[22] E. Bauer, P. Fischer, F. Marabelli, M. Ellerby, K. A. McEwen, B. Roessli, and M. T. Fernandes-Dias, Magnetic structures and bulk magnetic properties of $\mathrm{YbCu}_{4} \mathrm{M}, \mathrm{M}=\mathrm{Au}, \mathrm{Pd}$, Physica B 234-236, 676 (1997).

[23] M. A. Avila, S. L. Bud'ko, C. Petrovic, R. A. Ribeiro, P. C. Canfield, A. V. Tsvyashchenko, and L. N. Fomicheva, Synthesis and properties of $\mathrm{YbB}_{2}$, J. Alloys Compd. 358, 56 (2003).

[24] S. Ohara, T. Yamashita, Y. Mori, and I. Sakamoto, Transport and magnetic properties of new heavy-fermion antiferromagnet $\mathrm{YbNi}_{3} \mathrm{Al}_{9}$, J. Phys.: Conf. Ser. 273, 012048 (2011).

[25] C. Rossel, K. N. Yang, M. B. Maple, Z. Fisk, E. Zirngiebl, and J. D. Thompson, Strong electronic correlations in a new class of Yb-based compounds: $\mathrm{YbXCu}_{4}(X=\mathrm{Ag}, \mathrm{Au}, \mathrm{Pd})$, Phys. Rev. B 35, 1914 (1987).

[26] S. K. Dhar, C. Mitra, P. Manfrinetti, A. Palenzona, and P. Bonville, Magnetic behaviour of $\mathrm{Yb}_{2} \mathrm{Co}_{3} \mathrm{~T}_{9}(\mathrm{~T}=\mathrm{Ga}$ and $\mathrm{Al})$, Physica B 259-261, 150 (1999).

[27] O. Trovarelli, C. Geibel, B. Buschinger, R. Borth, S. Mederle, M. Grosche, G. Sparn, F. Steglich, O. Brosch, and L. Donnevert, Magnetic, transport, and thermal properties of $\mathrm{Yb}_{2} T_{3} X_{9}$ compounds ( $T=\mathrm{Rh}, \mathrm{Ir} ; X=\mathrm{Al}, \mathrm{Ga}$ ), Phys. Rev. B 60, 1136 (1999).

[28] S. L. Bud'ko, E. Morosan, and P. C. Canfield, Magnetic field induced non-Fermi-liquid behavior in $\mathrm{YbAgGe}$ single crystals, Phys. Rev. B 69, 014415 (2004).

[29] M. C. Bennett, P. Khalifah, D. A. Sokolov, W. J. Gannon, Y. Yiu, M. S. Kim, C. Henderson, and M. C. Aronson, A new unconventional antiferromagnet, $\mathrm{Yb}_{3} \mathrm{Pt}_{4}$, J. Magn. Magn. Mater. 321, 2021 (2009).

[30] B. Pollit, D. Dürkop, and P. Weidner, Magnetic ordering of mixed valent $\mathrm{Yb}_{3} \mathrm{Pd}_{4}$, J. Magn. Magn. Mater. 47-48, 583 (1985).

[31] C. Schank, G. Olesch, J. Köhler, U. Tegel, U. Klinger, J. Diehl, S. Klimm, G. Sparn, S. Horn, C. Geibel, and F. Steglich, YbNiAl: A new Yb-based heavy-fermion antiferromagnet, J. Magn. Magn. Mater. 140-144, 1237 (1995).

[32] A. Benoit, J. X. Boucherle, P. Convert, J. Flouquet, J. Palleau, and J. Schweizer, Magnetic structure of the compound $\mathrm{CeIn}_{3}$, Solid State Commun. 34, 293 (1980).

[33] J. Rossat-Mignod, P. Burlet, J. Villain, H. Bartholin, W. Tcheng$\mathrm{Si}$, D. Florence, and O. Vogt, Phase diagram and magnetic structures of CeSb, Phys. Rev. B 16, 440 (1977).

[34] G. Knebel, D. Aoki, G. Lapertot, B. Salce, J. Flouquet, T. Kawai, H. Muranaka, R. Settai, and Y. Ōnuki, High pressure phase diagram of the non-centrosymmetric antiferromagnet $\mathrm{CeCoGe}_{3}$, J. Phys. Soc. Jpn. 78, 074714 (2009).

[35] T. Nishioka, Y. Kawamura, T. Takesaka, R. Kobayashi, H. Kato, M. Matsumura, K. Kodama, K. Matsubayashi, and Y. Uwatoko,
Novel phase transition and the pressure effect in $\mathrm{YbFe}_{2} \mathrm{Al}_{10^{-}}$ type $\mathrm{Ce}_{2} \mathrm{Al}_{10}$ ( $T=\mathrm{Fe}$, Ru, Os), J. Phys. Soc. Jpn. 78, 123705 (2009).

[36] J. Kawabata, T. Takabatake, K. Umeo, and Y. Muro, Suppression of antiferromagnetic order and hybridization gap by electron and hole doping in the Kondo semiconductor $\mathrm{CeOs}_{2} \mathrm{Al}_{10}$, Phys. Rev. B 89, 094404 (2014).

[37] K. Kuga, Y. Karaki, Y. Matsumoto, Y. Machida, and S. Nakatsuji, Superconducting Properties of the Non-FermiLiquid System $\beta$-YbAlB 4 , Phys. Rev. Lett. 101, 137004 (2008).

[38] Y. Matsumoto, S. Nakatsuji, K. Kuga, Y. Karaki, N. Horie, Y. Shimura, T. Sakakibara, A. H. Nevidomskyy, and P. Coleman, Quantum criticality without tuning in the mixed valence compound $\beta$-YbAlB 4 , Science 331, 316 (2011).

[39] T. Tomita, K. Kuga, Y. Uwatoko, P. Coleman, and S. Nakatsuji, Strange metal without magnetic criticality, Science 349, 506 (2015).

[40] Y. Matsumoto, K. Kuga, T. Tomita, Y. Karaki, and S. Nakatsuji, Anisotropic heavy-Fermi-liquid formation in valence-fluctuating $\alpha-\mathrm{YbAlB}_{4}$, Phys. Rev. B 84, 125126 (2011).

[41] K. Kuga, Y. Matsumoto, M. Okawa, S. Suzuki, T. Tomita, K. Sone, Y. Shimura, T. Sakakibara, D. Nishio-Hamane, Y. Karaki et al., Quantum valence criticality in a correlated metal, Sci. Adv. 4, eaao3547 (2018).

[42] S. Watanabe and K. Miyake, Quantum Valence Criticality as an Origin of Unconventional Critical Phenomena, Phys. Rev. Lett. 105, 186403 (2010).

[43] M. Okawa, M. Matsunami, K. Ishizaka, R. Eguchi, M. Taguchi, A. Chainani, Y. Takata, M. Yabashi, K. Tamasaku, Y. Nishino et al., Strong Valence Fluctuation in the Quantum Critical Heavy Fermion Superconductor $\beta$-YbAlB 4 : A Hard X-Ray Photoemission Study, Phys. Rev. Lett. 104, 247201 (2010).

[44] T. Tomita, K. Kuga, Y. Uwatoko, and S. Nakatsuji, High pressure measurements of the resistivity of $\beta-\mathrm{YbAlB}_{4}$, J. Phys.: Conf. Ser. 592, 012019 (2015).

[45] T. Tomita, K. Kuga, Y. Uwatoko, and S. Nakatsuji, Pressureinduced magnetic transition exceeding $30 \mathrm{~K}$ in the Yb-based heavy-fermion $\beta$-YbAlB 4 , Phys. Rev. B 94, 245130 (2016).

[46] K. Kuga, G. Morrison, L. R. Treadwell, J. Y. Chan, and S. Nakatsuji, Magnetic order induced by Fe substitution of Al site in the heavy-fermion systems $\alpha-\mathrm{YbAlB}_{4}$ and $\beta$-YbAlB 4 , Phys. Rev. B 86, 224413 (2012).

[47] K. Kuga, S. Suzuki, and S. Nakatsuji, Two magnetic phases in $\alpha-\mathrm{YbAl}_{1-x} \mathrm{Fe}_{x} \mathrm{~B}_{4}$, JPS Conf. Proc. 3, 012013 (2014).

[48] T. T. Terashima, Y. H. Matsuda, K. Kuga, S. Suzuki, Y. Matsumoto, S. Nakatsuji, A. Kondo, K. Kindo, N. Kawamura, M. Mizumaki, and T. Inami, X-ray absorption spectroscopy in the heavy Fermion compound $\alpha-\mathrm{YbAlB}_{4}$ at high magnetic fields, J. Phys. Soc. Jpn. 84, 114715 (2015).

[49] P. G. de Gennes, Sur les propriétés des métaux des terres rares, C. r. hebd. séances Acad. Sci. 247, 1836 (1958).

[50] T. Mori, H. Borrmann, S. Okada, K. Kudou, A. Leithe-Jasper, U. Burkhardt, and Y. Grin, Crystal structure, chemical bonding, electrical transport, and magnetic behavior of $\mathrm{TmAlB}_{4}$, Phys. Rev. B 76, 064404 (2007).

[51] T. Mori, K. Kudou, T. Shishido, and S. Okada, f-electron dependence of the physical properties of $\mathrm{REAlB}_{4}$; an $\mathrm{AlB}_{2}$ type analogous "tiling" compound, J. Appl. Phys. 109, 07E111 (2011). 
[52] A. V. Goltsev and M. M. Abd-Elmeguid, Origin of the pressure dependence of the Kondo temperature in Ce- and Yb-based heavy-fermion compounds, J. Phys.: Condens. Matter 17, S813 (2005).

[53] J. Flouquet and H. Harima, Heavy fermion material: Ce versus Yb case, arXiv:0910.3110 (English); Kotai Butsuri (Japanese) 47, 2, 1 (2012).

[54] Y. Sakaguchi, S. Ikeda, K. Kuga, S. Suzuki, S. Nakatsuji, N. Hirao, Y. Ohishi, and H. Kobayashi, Pressure-induced local structural changes in heavy Fermion $\beta$-YbAlB 4 , J. Phys. Soc. Jpn. 85, 023602 (2016).

[55] A. H. Nevidomskyy and P. Coleman, Layered Kondo Lattice Model for Quantum Critical $\beta$-YbAlB 4 , Phys. Rev. Lett. 102, 077202 (2009).

[56] A. Ramires, P. Coleman, A. H. Nevidomskyy, and A. M. Tsvelik, $\beta$-YbAlB ${ }_{4}$ : A Critical Nodal Metal, Phys. Rev. Lett. 109, 176404 (2012).

[57] T. Nakano, K. Fujita, S. Murayama, K. Hoshi, M. Hedo, and Y. Uwatoko, Resistivity of $\mathrm{Ce}\left(\mathrm{Ru}_{0.85} \mathrm{Rh}_{0.15}\right)_{2} \mathrm{Si}_{2}$ under pressure, Physica B 329-333, 532 (2003).

[58] T. Kiss, F. Kanetaka, T. Yokoya, T. Shimojima, K. Kanai, S. Shin, Y. Onuki, T. Togashi, C. Zhang, C. T. Chen, and S. Watanabe, Photoemission Spectroscopic Evidence of Gap Anisotropy in an $f$-Electron Superconductor, Phys. Rev. Lett. 94, 057001 (2005).

[59] T. Shimojima, K. Okazaki, and S. Shin, Low-temperature and high-energy-resolution laser photoemission spectroscopy, J. Phys. Soc. Jpn. 84, 072001 (2015).

[60] D. Ehm, S. Hüfner, F. Reinert, J. Kroha, P. Wölfle, O. Stockert, C. Geibel, and H. v. Löhneysen, High-resolution photoemission study on low- $T_{K}$ Ce systems: Kondo resonance, crystal field structures, and their temperature dependence, Phys. Rev. B 76, 045117 (2007).

[61] See the Appendix E for further information.

[62] C. Bareille, S. Suzuki, M. Nakayama, K. Kuroda, A. H. Nevidomskyy, Y. Matsumoto, S. Nakatsuji, T. Kondo, and S. Shin, Kondo hybridization and quantum criticality in $\beta-\mathrm{YbAlB}_{4}$ by laser ARPES, Phys. Rev. B 97, 045112 (2018).

[63] A. C. Hewson, The Kondo Problem to Heavy Fermions (Cambridge University Press, Cambridge, 1993).

[64] A. Generalov, D. A. Sokolov, A. Chikina, Y. Kucherenko, V. N. Antonov, L. V. Bekenov, S. Patil, A. D. Huxley, J. W. Allen, K. Matho et al., Insight into the temperature dependent properties of the ferromagnetic Kondo lattice YbNiSn, Phys. Rev. B 95, 184433 (2017).

[65] T. Takabatake, F. Teshima, H. Fujii, S. Nishigori, T. Suzuki, T. Fujita, Y. Yamaguchi, J. Sakurai, and D. Jaccard, Formation of an anisotropic energy gap in the valence-fluctuating system of CeNiSn, Phys. Rev. B 41, 9607 (1990).

[66] S. K. Malik and D. T. Adroja, Evidence of pseudogap formation in a new valence-fluctuating compound: CeRhSb, Phys. Rev. B 43, 6277 (1991)

[67] H. Kumigashira, T. Sato, T. Yokoya, T. Takahashi, S. Yoshii, and M. Kasaya, Spectral Evidence for Pseudogap Formation in Kondo Insulators CeRhAs and CeRhSb, Phys. Rev. Lett. 82, 1943 (1999).

[68] Y. Muro, J. Kajino, K. Umeo, K. Nishimoto, R. Tamura, and T. Takabatake, Structural modification and metamagnetic anomaly in the ordered state of $\mathrm{CeOs}_{2} \mathrm{Al}_{10}$, Phys. Rev. B 81, 214401 (2010).
[69] T. Takesaka, K. Oe, R. Kobayashi, Y. Kawamura, T. Nishioka, H. Kato, M. Matsumura, and K. Kodama, Semiconducting behavior in $\mathrm{CeFe}_{2} \mathrm{Al}_{10}$ and $\mathrm{CeRu}_{2} \mathrm{Al}_{10}$ single crystals, J. Phys.: Conf. Ser. 200, 012201 (2010)

[70] S.-i. Kimura, T. Iizuka, H. Miyazaki, A. Irizawa, Y. Muro, and T. Takabatake, Electronic-Structure-Driven Magnetic Ordering in a Kondo Semiconductor $\mathrm{CeOs}_{2} \mathrm{Al}_{10}$, Phys. Rev. Lett. 106, 056404 (2011).

[71] T. Yoshida, T. Ohashi, and N. Kawakami, Effects of conduction electron correlation on heavy-Fermion systems, J. Phys. Soc. Jpn. 80, 064710 (2011).

[72] S. A. Werner, A. Arrott, and H. Kendrick, Temperature and magnetic-field dependence of the antiferromagnetism in pure chromium, Phys. Rev. 155, 528 (1967).

[73] S. Suzuki, T. Tomita, Y. Shimura, K. Kuga, Y. Matsumoto, and S. Nakatsuji, High magnetic transition temperature and semiconductor like transport properties of Mn-doped $\alpha-\mathrm{YbAlB}_{4}, \mathrm{~J}$. Phys.: Conf. Ser. 683, 012009 (2016).

[74] K.-H. Müller and V. N. Narozhnyi, Interaction of superconductivity and magnetism in borocarbide superconductors, Rep. Prog. Phys. 64, 943 (2001).

[75] C. L. Seaman, N. R. Dilley, M. C. de Andrade, J. Herrmann, M. B. Maple, and Z. Fisk, Superconductivity and magnetism in the Heusler alloys $M \mathrm{Pd}_{2} \mathrm{~Pb}(M=$ rare earth, Th, and $\mathrm{U})$, Phys. Rev. B 53, 2651 (1996).

[76] P. G. Pagliuso, J. D. Thompson, M. F. Hundley, J. L. Sarrao, and Z. Fisk, Crystal structure and low-temperature magnetic properties of $R_{m} M \mathrm{In}_{3 m+2}$ compounds $(\mathrm{M}=\mathrm{Rh}$ or $\mathrm{Ir} ; \mathrm{m}=1,2$ $\mathrm{R}=$ Sm or Gd), Phys. Rev. B 63, 054426 (2001).

[77] T. Ishiga, T. Wakita, R. Yoshida, H. Okazaki, K. Tsubota, M. Sunagawa, K. Uenaka, K. Okada, H. Kumigashira, M. Oshima, K. Yutani, Y. Muro, T. Takabatake, Y. Muraoka, and T. Yokoya, Electronic structures of $\mathrm{Ce}_{2} \mathrm{Al}_{10}(M=\mathrm{Fe}, \mathrm{Ru}$, and Os) studied by soft $\mathrm{x}$-ray resonant and high-resolution photoemission spectroscopiest, J. Phys. Soc. Jpn. 83, 094717 (2014).

[78] L. H. Tjeng, S.-J. Oh, E.-J. Cho, H.-J. Lin, C. T. Chen, G.-H Gweon, J.-H. Park, J. W. Allen, T. Suzuki, M. S. Makivić, and D. L. Cox, Temperature dependence of the Kondo resonance in $\mathrm{YbAl}_{3}$, Phys. Rev. Lett. 71, 1419 (1993).

[79] Y. Takeda, M. Arita, M. Higashiguchi, K. Shimada, H Namatame, M. Taniguchi, F. Iga, and T. Takabatake, Highresolution photoemission study of the temperature-dependent $c-f$ hybridization gap in the Kondo semiconductor $\mathrm{YbB}_{12}$, Phys. Rev. B 73, 033202 (2006).

[80] A. Shigemoto, J. Yamaguchi, A. Sekiyama, S. Imada, A Yamasaki, A. Irizawa, T. Ukawa, T. Muro, F. Iga, T. Takabatake, and S. Suga, Observation of bulk electronic states of Kondo semiconductor $\mathrm{YbB}_{12}$ by high-resolution soft $\mathrm{x}$-ray photoemission spectroscopy, J. Electron Spectrosc. 156-158, 472 (2007).

[81] T. Mutou and D. S. Hirashima, Gap formation in the symmetric periodic Anderson model in infinite dimensions, J. Phys. Soc. Jpn. 63, 4475 (1994).

[82] T. A. Costi and N. Manini, Low-energy scales and temperaturedependent photoemission of heavy Fermions, J. Low Temp. Phys. 126, 835 (2002).

[83] V. Madhavan, W. Chen, T. Jamneala, M. F. Crommie, and N. S. Wingreen, Tunneling into a single magnetic atom: Spectroscopic evidence of the Kondo resonance, Science 280, 567 (1998). 
[84] A. R. Schmidt, M. H. Hamidian, P. Wahl, F. Meier, A. V. Balatsky, J. D. Garrett, T. J. Williams, G. M. Luke, and J. C. Davis, Imaging the Fano lattice to 'hidden order' transition in $\mathrm{URu}_{2} \mathrm{Si}_{2}$, Nature (London) 465, 570 (2010).

[85] S. Ernst, S. Kirchner, C. Krellner, C. Geibel, G. Zwicknagl, F. Steglich, and S. Wirth, Emerging local Kondo screening and spatial coherence in the heavy-fermion metal $\mathrm{YbRh}_{2} \mathrm{Si}_{2}$, Nature (London) 474, 362 (2011).

[86] P. Aynajian, E. H. da Silva Neto, A. Gyenis, R. E. Baumbach, J. D. Thompson, Z. Fisk, E. D. Bauer, and A. Yazdani, Visualizing heavy fermions emerging in a quantum critical Kondo lattice, Nature (London) 486, 201 (2012).

[87] A. C. Hewson, The Kondo Problem to Heavy Fermions (Cambridge University Press, Cambridge, 1993).
[88] M. Maltseva, M. Dzero, and P. Coleman, Electron Cotunneling into a Kondo Lattice, Phys. Rev. Lett. 103, 206402 (2009).

[89] P. Wölfle, Y. Dubi, and A. V. Balatsky, Tunneling into Clean Heavy Fermion Compounds: Origin of the Fano Line Shape, Phys. Rev. Lett. 105, 246401 (2010).

[90] D. A. Tompsett, Z. P. Yin, G. G. Lonzarich, and W. E. Pickett, Role of crystal symmetry in the magnetic instabilities of $\beta-\mathrm{YbAlB}_{4}$ and $\alpha-\mathrm{YbAlB}_{4}$, Phys. Rev. B 82, 235101 (2010).

[91] E. C. T. O'Farrell, D. A. Tompsett, S. E. Sebastian, N. Harrison, C. Capan, L. Balicas, K. Kuga, A. Matsuo, K. Kindo, M. Tokunaga, S. Nakatsuji, G. Csányi, Z. Fisk, and M. L. Sutherland, Role of $f$ Electrons in the Fermi Surface of the Heavy Fermion Superconductor $\beta$-YbAlB 4 , Phys. Rev. Lett. 102, 216402 (2009). 